\title{
Analysis of pion elliptic flows and HBT interferometry in a granular quark-gluon plasma droplet model
}

\author{
Wei-Ning Zhang ${ }^{1,2,3}$, Yan-Yu Ren ${ }^{2}$, and Cheuk-Yin Wong ${ }^{3,4}$ \\ ${ }^{1}$ Department of Physics, Dalian University of Technology, Dalian, Liaoning 116024, P. R. China \\ ${ }^{2}$ Department of Physics, Harbin Institute of Technology, Harbin, Heilongjiang 150006, P. R. China \\ ${ }^{3}$ Physics Division, Oak Ridge National Laboratory, Oak Ridge, TN 37831, U.S.A. \\ ${ }^{4}$ Department of Physics, University of Tennessee, Knoxville, TN 37996, U.S.A.
}

(Dated: April 30, 2018)

\begin{abstract}
In many simulations of high-energy heavy-ion collisions on an event-by-event analysis, it is known that the initial energy density distribution in the transverse plane is highly fluctuating. Subsequent longitudinal expansion will lead to many longitudinal tubes of quark-gluon plasma which have tendencies to break up into many spherical droplets because of sausage instabilities. We are therefore motivated to use a model of quark-gluon plasma granular droplets that evolve hydrodynamically to investigate pion elliptic flows and Hanbury-Brown-Twiss interferometry. We find that the data of pion transverse momentum spectra, elliptic flows, and HBT radii in $\sqrt{s_{N N}}=200 \mathrm{GeV} \mathrm{Au}+$ $\mathrm{Au}$ collisions at RHIC can be described well by an expanding source of granular droplets with an anisotropic velocity distribution.
\end{abstract}

PACS numbers: 25.75.-q, 25.75.Nq, 25.75.Gz

\section{INTRODUCTION}

Recently, there has been much progress in our understanding of the process of nucleus-nucleus collisions at RHIC 1, 2, 3, 4, 5, 6]. Hydrodynamical calculations agree well with the RHIC $v_{2}$ data of the elliptic flow at low transverse momentum $p_{T}<2 \mathrm{GeV}$ [7, [8, 9, 10, 11]. However, they cannot predict the saturation of $v_{2}$ at higher $p_{T}$, $8,9,10,11$ and the RHIC HBT puzzle of $R_{\text {out }} / R_{\text {side }} \approx 1$ [1, 13, 14, 15]. The HBT puzzle is contrary to many earlier theoretical expectations 16, 17, 18, 19, 20, 21. Various models have been put forth to explain the HBT puzzle 22, 23, 24, 25, 26, 27, 28, 29, 30, 31, 32, 33, 34]. There have also been many attempts to provide a consistent explanation for both the elliptic flow and HBT measurements at RHIC 35, 36, 37, 38, 39, 40, 41].

In Ref. 28], a granular particle-emitting source of quark-gluon plasma (QGP) droplets evolving hydrodynamically was put forth to explain the RHIC HBT puzzle. The suggestion was based on the observation that in the hydrodynamical model [16, 42], the particle emission time scales with the initial radius of the droplet. Particles will be emitted earlier if the radius of the droplet is smaller, as in a source of many droplets. An earlier emission time will lead to a smaller extracted HBT radius $R_{\text {out }}$, while the extracted HBT radius $R_{\text {side }}$ is determined by the scale of the distribution of the droplets. As a result, the value of $R_{\text {out }}$ can be close to $R_{\text {side }}$ for a granular quark-gluon plasma source [28]. Further suggestions of using the single-event intensity interferometry to search for the signature for granular structures have also been presented [43, 44].

Motivated by the successes of our previous analysis of the HBT puzzle using a granular droplet model, we examine in this paper the theoretical basis for the possible occurrence of granular structure in the evolution of a quark- gluon plasma. In addition, we wish to refine the granular source model of Ref. [28] by considering more reasonable anisotropic velocity distributions of the droplets instead of a constant radial velocity assumed in [28]. We would like to investigate the pion elliptic flows as well as HBT radii as functions of the pion transverse momentum. The agreement both of the $v_{2}$ and HBT radii with experimental data concurrently will gives strong constrains for the granular model and its parameters and will provide a useful insight into the initial state and the evolution of the particle-emitting source produced in high-energy nucleus-nucleus collisions.

\section{GRANULAR INSTABILITY IN THE EVOLUTION OF THE QUARK-GLUON PLASMA}

Based on the recent results of high-energy heavy-ion collisions at RHIC, the matter (presumably QGP) produced in the collisions may be a strongly-coupled medium with a very high energy density $1,[2,3,4,[5,6]$. They are thermalized within about $1 \mathrm{fm} / \mathrm{c} 1,12,3,4,5,6]$. It is of interest to study its subsequent evolution and see whether granular structures may play a role in the spacetime development of the presumed quark-gluon plasma matter.

Although a granular structure was suggested earlier as the signature of a first-order phase transition [45], the occurrence of granular structure may not be limited to the occurrence of a first-order phase transition. There are additional effects which may lead to the dynamical formation of granular droplets.

Because the QGP is a strongly interacting dense medium, a surface tension arises at a boundary due to the presence of a strong interaction in the dense phase on one side of the boundary and the absence (or weakening) of the strong interaction with no density (or diminishing 
density) on the other side [46, 47, 48, 49, 50, 51, 52, 53]. This imbalance of the forces acting on different parts at the boundary leads to the surface tension, while the detail profile of the boundary may depend on the nature and the order of phase transition. Due to the presence of this surface tension, there may be instability against surface shape changes and bulk density oscillations.

\section{A. Transverse Density Fluctuations and Surface Granular Instability}

The quark gluon plasma that may be produced in the laboratory is spatially bounded by various boundaries. Furthermore, in many simulations of the heavy-ion collisions on an event-by-event basis, the initial transverse energy density is far from being uniform. It exhibits large transverse density fluctuations with a large peakto-valley ratio 29, 41, [54]. A large number of transverse density domains are clearly visible in the initial transverse density distribution of the produced matter in Fig. 21 of [54] or Fig. 4 of [41]. Because of these highly fluctuating initial transverse density distribution forming "lumps" in different regions, the probability for the occurrence of granular structure may be enhanced.

We can present an approximate scenario to discuss the evolution of matter with a large transverse density fluctuations, in a central collision of two heavy and equal nuclei of radius $R$. Because of Lorentz contraction, the longitudinal length $R / \gamma$ of the produced matter is much smaller than the transverse length, which is of the order $R$. Initially as depicted in Fig. 1(a), density fluctuations in the transverse plane manifest as a number of transverse lumps characterized by a radius $r_{d 0}$, which one can also see from the results of [54] and [41]. From such an initial data, we can consider the evolution according to Landau hydrodynamics 55], which gives a good description of the longitudinal distribution of the produced particles [56, 57]. In such a description, because the initial longitudinal length is much smaller than the transverse length, the hydrodynamical force per unit volume in the longitudinal direction, $-\partial P / \partial z$, is much greater than the hydrodynamical force per unit volume in the transverse direction, $-\partial P / \partial \rho$. The subsequent expansion in the longitudinal direction proceeds much faster than in the transverse direction, as pointed out by Landau and Belenkii [55]. The expanding matter develops into tubes of radius $r_{d 0}$ [Fig. 1(b)] and the density of matter in the tube decreases as a function of the proper time.

As is well known, a long tube of matter is unstable against "sausage" perturbations that tend to make the tube narrow in some regions and thicker in other regions. The sausage instability leads to the break-up of the long tube of matter into many approximately equal spherical droplets, as in the break-up of toroidal liquid droplets 58.

We can examine the sausage instability for a cylinder with a length $L$ and a radius $r_{d 0}$, with $L>>r_{d 0}$. We
$\rho$
(a) Initial configuration

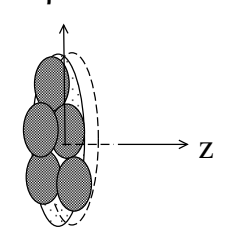

(b) Shortly afterwards

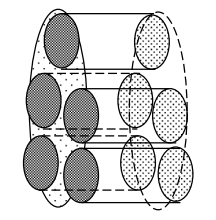

(c) Later afterwards

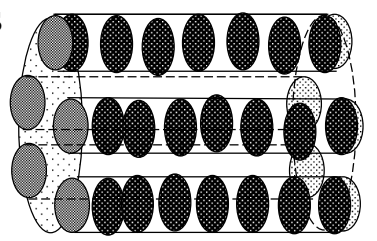

FIG. 1: Schematic description of the matter distribution under a rapid longitudinal expansion in the $z$-direction, for a highly fluctuating initial transverse density distribution [Fig. $1 a]$. The system evolves into many longitudinal tubes at Fig. $1 b$ and later at Fig. 1c. Sausage instabilities lead to the production of droplets along the longitudinal tubes as depicted in Fig. 1c.

consider "sausage" perturbations $a_{n}$ about the cylinder of matter,

$$
r_{d}(z)=\tilde{r}_{d 0}\left\{1+\sum_{n=\mathrm{odd}} a_{n} \sin (n \pi z / L)\right\} .
$$

In order to have a positive slope $d r_{d}(z) / d z$ at $z=0$ and a negative slope at $z=L$, we need to limit ourselves to perturbations with odd values of $n$ and $a_{n}>0$. By the condition of volume conservation, the quantity $\tilde{r}_{d 0}$ is

$\tilde{r}_{d 0}=r_{d 0}\left\{1-\sum_{n=\text { odd }} \frac{2 a_{n}}{n \pi}-\frac{1}{4} \sum_{n=\text { odd }} a_{n}^{2}+\frac{3}{2}\left(\sum_{n=\text { odd }} \frac{2 a_{n}}{n \pi}\right)^{2}\right\}$

The surface energy of the cylinder is then

$$
E_{s}=\sigma 2 \pi r_{d 0} L\left\{1-\frac{1}{4} \sum_{n=\text { odd }} a_{n}{ }^{2}+\frac{1}{2}\left(\sum_{n=\text { odd }} \frac{2 a_{n}}{n \pi}\right)^{2}\right\} .
$$

The cylinder is unstable against perturbations of order $n$ if $d^{2} E_{s} / d a_{n}^{2}$ is negative. We can evaluate $d^{2} E_{s} / d a_{n}^{2}$ and obtain

$$
d^{2} E_{s} / d a_{n}^{2}=\sigma 2 \pi r_{d 0} L\left\{-1 / 2+(2 / n \pi)^{2}\right\} .
$$

Thus, $d^{2} E_{s} / d a_{n}^{2}$ is negative for all odd integer values of $n$. As an unrestrained growth of the perturbation of order $n$ leads the formation of $(n+1) / 2$ droplets, we find that the cylinder is unstable against perturbations leading to the formation of $1,2,3, \ldots$ approximately 
equal droplets. Thus, when the length $L$ of the tubes are stretched, sausage (granular) instability will develop and the long tube will fragment into approximately equalsize droplets, as depicted in Fig. 2c. Large density fluctuations in the transverse direction, together with the surface tension effects, favor the formation of granular droplets.

In our simplified description, we have ignored the effects of the perturbation on the variation of the transverse surfaces at the two ends of the cylinder. A refinement on the description of the end surfaces will not modify significantly the question of the instability of the cylindrical matter, for cylinders with $L$ substantially greater than $r_{d 0}$.

In conclusion, we find from the above analysis that the occurrence of granular droplets may be more common than previously thought. It is of great interest to explore the subsequent dynamics in a granular model for the description of various observables measured in high-energy heavy-ion collisions. This is particularly relevant because a granular droplet model can explain the occurrence of early emission of particles and a small value of $R_{\text {out }}[28]$.

\section{B. Bulk density oscillations due to surface tension interactions}

To carry out the analysis of the effects of surface tension on the stability of density oscillations, we consider the following simple model of the surface tension, as represented by a finite-range interaction. The equation of hydrodynamics without a finite-range interaction is [59]

$$
[\epsilon+p] u^{k} \frac{\partial u_{i}}{\partial x^{k}}=-\frac{\partial p}{\partial x^{i}}-u_{i} u^{k} \frac{\partial p}{\partial x^{k}},
$$

where $\epsilon$ and $p$ are the energy density and pressure. In this hydrodynamical equation, interactions between constituents leading to bulk properties of the plasma have already been included into the characterization of the pressure $p$ and the energy density $\epsilon$. What remains is the effective residual finite-range interaction $v\left(\mathbf{r}-\mathbf{r}^{\prime}\right)$ that can lead to the surface tension. In the presence of this finite-range residual interaction, the above can be generalized to be [60]

$$
\begin{aligned}
{[\epsilon(\mathbf{r})} & +p(\mathbf{r})] u^{k}(\mathbf{r}) \frac{\partial u_{i}(\mathbf{r})}{\partial x^{k}}=-\frac{\partial p(\mathbf{r})}{\partial x^{i}}-u_{i}(\mathbf{r}) u^{k}(\mathbf{r}) \frac{\partial p(\mathbf{r})}{\partial x^{k}} \\
& -\int d \mathbf{r}^{\prime} \frac{\epsilon(\mathbf{r})}{W} \frac{\epsilon\left(\mathbf{r}^{\prime}\right)}{W} \nabla_{i} v\left(\mathbf{r}-\mathbf{r}^{\prime}\right),
\end{aligned}
$$

where $W$ is the average energy per particle which may be temperature-dependent, and $\epsilon(\mathbf{r}) / W$ gives the number density $n(\mathbf{r})$ of the constituents at $\mathbf{r}$.

We choose to represent the effect of the surface tension in terms of an effective residual interaction of the following form as used in [47],

$$
v(r)=\alpha_{r}\left(1-\frac{\mu r}{2}\right) \frac{e^{-\mu r}}{r},
$$

where $\alpha_{r}>0$ and $1 / \mu$ are the strength and the range of the residual interaction that leads to the surface tension. As a residual interaction, $v(r)$ has been chosen such that the contribution of the interaction to the total energy of a system with a uniform density is zero and is positive and proportional to the surface area in the surface region.

To carry out the stability analysis, we can envisage that by solving the hydrodynamical equation (5) without the surface interaction, one obtains a comparatively slowvarying density distribution $\epsilon_{0}(\mathbf{r}, t)$ with a local average $\bar{\epsilon}_{0}=\left\langle\epsilon_{0}(\mathbf{r}, t)\right\rangle$. Then following the derivations of the equation of sound waves [59], the perturbation $\delta \epsilon(\mathbf{r}, t)=$ $\epsilon(\mathbf{r}, t)-\epsilon_{0}(\mathbf{r}, t)$ obeys the wave equation

$$
\begin{aligned}
\frac{\partial^{2} \delta \epsilon(\mathbf{r}, \mathbf{t})}{\partial t^{2}} \approx & c_{s}^{2} \nabla^{2} \delta \epsilon(\mathbf{r}, \mathbf{t})+\nabla_{\mathbf{r}} \cdot \int d \mathbf{r}^{\prime}\left[\left(\delta \epsilon(\mathbf{r}, t) \epsilon\left(\mathbf{r}^{\prime}, t\right)\right.\right. \\
& \left.+\epsilon(\mathbf{r}, t)\left(\delta \epsilon\left(\mathbf{r}^{\prime}, t\right)\right)\right] \nabla_{\mathbf{r}} v\left(\mathbf{r}-\mathbf{r}^{\prime}\right) / W^{2}
\end{aligned}
$$

where $c_{s}$ is the speed of sound. We consider density perturbations of the type

$$
\delta \epsilon(\mathbf{r}, t)=e^{i \mathbf{k} \cdot \mathbf{r}-i \omega t} .
$$

Representing the spatial variation of $\epsilon_{0}(\mathbf{r}, t)$ by its average $\bar{\epsilon}_{0}$, we obtain from Eq. (8)

$$
\omega^{2}=c_{s}^{2} f(\mathbf{k}) \mathbf{k}^{2},
$$

for points in the interior of the medium. Here, $f(\mathbf{k})$ is

$$
f(\mathbf{k})=1-\frac{4 a \mu^{2} \mathbf{k}^{2}}{\left(\mathbf{k}^{2}+\mu^{2}\right)^{2}},
$$

where the second term of $f(\mathbf{k})$ arises from the surface tension interaction $v(r)$, and $a$ is the granular stability number defined as

$$
a=\frac{\bar{\epsilon}_{0} \pi \alpha_{r}}{W^{2} \mu^{2} c_{s}^{2}} .
$$

Equations (10) and (11) indicates that when $f(k)$ becomes negative, the system is unstable against a density perturbation of wave number $k$. We plot in Fig. 2 the function $f(k)$ as a function of $k / \mu$. As one observes, the minimum of $f(k)$ is located at $k / \mu=1$ for which the characteristic wave length of the oscillation $1 / k$ is equal to the range of the interaction $1 / \mu$. The function $f(k)$ becomes negative when $a \geq 1$ and it is therefore appropriate to call $a$ the granular stability number. When the granular stability number $a$ is greater than unity, the system is unstable against perturbations with wave numbers $k$ in the interval $k_{-} \leq k \leq k_{+}$, with $k_{ \pm}$given by

$$
\mathbf{k}_{ \pm}^{2}=\left\{2 a-1 \pm \sqrt{(2 a-1)^{2}-1}\right\} \mu^{2} .
$$

We note from Eq. (12) that the condition $a \geq 1$ for the occurrence of granular instability happens when the surface tension interaction strength $\alpha_{r}$ is large. It will be useful to relate $\alpha_{r}$ to the surface tension coefficient 


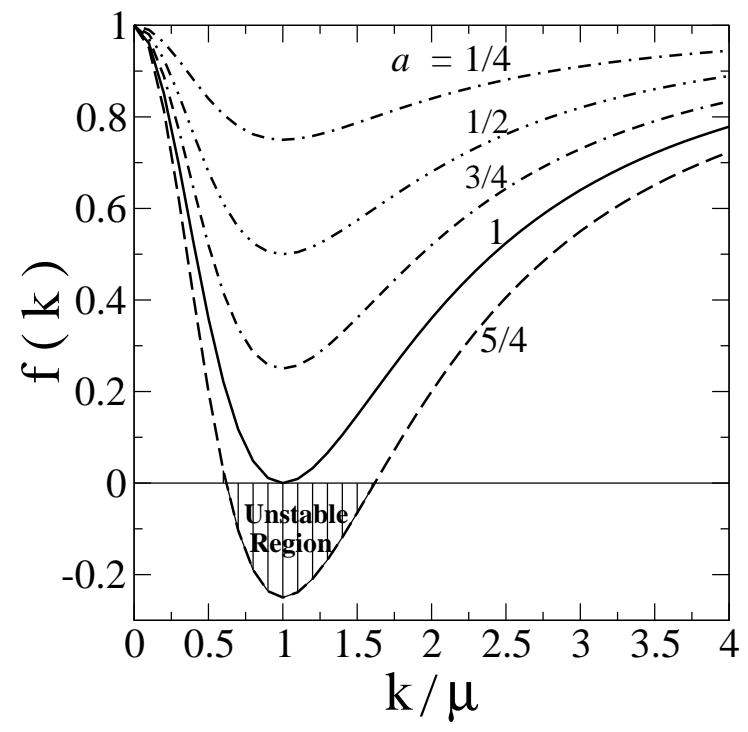

FIG. 2: $f(\mathbf{k})$ curves for different values of $a$. The expansion is unstable in the shadowy region.

$\sigma$. For such a purpose, we study the energy of a system with a numbers density profile given by

$$
n(\mathbf{r})=n_{\text {out }}+\frac{n_{0}-n_{\text {out }}}{1+e^{z / a}}
$$

where $n_{0}\left(>n_{\text {out }}\right)$ is the number density in the interior dense region of a surface and $n_{\text {out }}$ is the number density in the outer dilute region. The energy of the system due to the residual interaction of Eq. (17) is

$$
E=\frac{1}{2} \int d \boldsymbol{\rho} d z n(\mathbf{r}) \int d \boldsymbol{\rho}^{\prime} d z^{\prime} n\left(\mathbf{r}^{\prime}\right) v\left(\mathbf{r}-\mathbf{r}^{\prime}\right) .
$$

We can introduce $V(z)$ as

$$
V(z)=\int d \boldsymbol{\rho}^{\prime} d z^{\prime} n\left(\mathbf{r}^{\prime}\right) v\left(\mathbf{r}-\mathbf{r}^{\prime}\right)
$$

After integration the $\rho^{\prime}$ coordinates, we find

$$
\begin{aligned}
V(z) & =\frac{\pi \alpha_{r}}{\mu} \int_{-\infty}^{\infty} d z^{\prime} n\left(z^{\prime}\right) \\
& \times\left\{e^{-\mu\left|z-z^{\prime}\right|}-\mu\left|z-z^{\prime}\right| e^{-\mu\left|z-z^{\prime}\right|}\right\} .
\end{aligned}
$$

From Eqs. (15)-(17), the surface tension coefficient is therefore given by

$$
\begin{aligned}
\sigma & \equiv d E / d \boldsymbol{\rho} \\
& =\frac{\alpha_{r} \pi}{2 \mu} \int_{-\infty}^{\infty} d z n(z) \int_{-\infty}^{\infty} d z^{\prime} n\left(z^{\prime}\right) \\
& \times\left\{e^{-\mu\left|z-z^{\prime}\right|}-\mu\left|z-z^{\prime}\right| e^{-\mu\left|z-z^{\prime}\right|}\right\}
\end{aligned}
$$

which gives a relation between $\sigma$ and $\alpha_{r}$ for a general density profile. For the simple case of a sharp boundary with $n(z)=n_{0} \theta(-z)$, the above equation gives the relationship

$$
\sigma=\frac{n_{0}^{2} \pi \alpha_{r}}{2 \mu^{3}}
$$

The surface tension coefficient increases with $\alpha_{r} / \mu^{3}$ and the square of the number density. As $n_{0}$ varies with temperature as $T^{3}$ while $\alpha_{r}$ and $\mu$ are relatively slowly varying functions of $T$, the surface tension varies with temperature approximately as $T^{6}$. When the surface is described by a sharp surface profile, the granular stability number $a$ is related to the surface tension coefficient $\sigma$ by

$$
a=\frac{2 \mu \sigma}{\epsilon_{0} c_{s}^{2}} .
$$

In quenched QCD lattice gauge calculations, the surface tension coefficient $\sigma\left(T_{c}\right)$ has been calculated for $T=T_{c}$, and $\sigma\left(T_{c}\right) / T_{r}^{3}$ was found to range from 0.016 to $0.092[50,51,52,53]$. If we use such a value of the surface tension coefficient to evaluate the granular stability number $a$ using Eq. (12), the quantity $a$ will have the value from 0.024 to 0.140 and the system is stable against density oscillations of the type discussed here. However, the medium considered by the quenched QCD approximation consists of gluons without dynamical quarks. As is well known, the presence of dynamical quarks has important influences on many thermodynamical properties of the plasma. The phase transition temperature is altered from $269 \mathrm{MeV}$ for quenched QCD without dynamical quarks to $154 \mathrm{MeV}$ for full QCD with 3 flavors of quarks [61], whereas the surface tension coefficient $\sigma$ depends on the temperature approximately as $T^{6}$. The number density of a quark-gluon plasma with three flavors of quarks is about two times the number density of the plasma without dynamical quarks, while the surface tension coefficient varies as the square of the number density. The surface tension coefficient $\sigma$ calculated in quenched QCD at $T=T_{c}$ may be quite different from that calculated in full QCD at higher temperatures. It will be of interest to evaluate the surface tension coefficient in full QCD and study its temperature dependence in order to find out whether the quark-gluon plasma is stable against bulk density oscillations with wave lengths close the the range of the interaction discussed here.

The above discussions was carried out in the linearized perturbative theory of slow hydrodynamical motion. We envisage that the initial QGP matter is highly compressed in the longitudinal direction and the subsequent longitudinal expansion will be rather rapid and nonlinear in the density changes. In this respect, it is of interest to note that in previous calculations in full hydrodynamics for the rapid expansion of a nuclear system with a finite range interaction, the one-dimensional fragmentation of the density into one-dimensional "lumps' occur, as shown in the left panel of Fig. 2 of Ref. 62]. If these hydrodynamical calculations can be a useful analogue, the analogous occurrence of granular droplets in 
the bulk expansion of the quark-gluon plasma may be possible.

\section{MODEL OF GRANULAR QGP DROPLETS}

Based on the above discussions, we would like to use a model of granular quark-gluon plasma droplets to examine the dynamical evolution of the system. We assume that the system consists of $N_{d}$ droplets of radius $r_{d}$ initially distributed in a short cylinder of length $2 \mathcal{R}_{z}$ along the beam direction ( $z$ direction) as in Fig. $2 c$, with an initial transverse spatial distribution up to a radius $\mathcal{R}_{t}$,

$$
\begin{aligned}
\frac{d P_{d}}{2 \pi \rho d \rho d z} \propto & {\left[1-\exp \left(-\rho^{2} / \Delta \mathcal{R}_{t}^{2}\right)\right] } \\
& \times \theta\left(\mathcal{R}_{t}-\rho\right) \theta\left(\mathcal{R}_{z}-|z|\right),
\end{aligned}
$$

where $\rho=\sqrt{x^{2}+y^{2}}$ and $z$ are the coordinates of the center of a droplet. The parameter $\Delta \mathcal{R}_{t}$ describes a shelltype radial distribution which may arise from the dynamics of the transverse expansion. A naive blast-wave-type expansion with a constant radial velocity would leave a void along the central transverse axis at $\rho=0$. Instead of a constant transverse velocity, we shall find later that the best parameters that describe the experimental data suggests a transverse velocity profile $\left(\beta_{d}\right)_{T} \sim \rho^{0.42}$. From the equation of continuity, the density would change approximately by

$$
\frac{d n}{d t} \sim-n \frac{1}{\rho} \frac{d}{d \rho} \rho\left(\beta_{d}\right)_{T} \propto-1.42 \frac{n}{\rho^{0.58}} .
$$

Thus the density decreases more rapidly near $\rho \sim 0$ and there is a tendency to form a shell-type radial distribution. The shell-type distribution of the droplets may also arise from the shadowing of detected particles originating from the central region of $\rho \sim 0$. The HBT radii as a function of the average pion transverse momentum of a pion pair turns out to be slightly sensitive to this parameter of $\Delta \mathcal{R}_{t}$.

Because of the early thermalization and the anisotropic pressure gradient, the droplets will acquire anisotropic initial velocities. We assume that the velocity of a droplet depends on the initial coordinates of the droplet center, $\left(r_{1}, r_{2}, r_{3}\right)=(x, y, z)$, in the form

$$
\left(\boldsymbol{\beta}_{d}\right)_{i}=a_{i} \operatorname{sign}\left(r_{i}\right)\left(\frac{\left|r_{i}\right|}{\mathcal{R}_{i}}\right)^{b_{i}}, \quad i=1,2, \text { and } 3,(23)
$$

where $a_{i}$ is describes the magnitude of the anisotropic expansion, $\operatorname{sign}\left(r_{i}\right)$ denotes the sign of $r_{i}$, and $b_{i}\left(b_{T}, b_{z}\right)$ are the exponential power parameter that describes the variation of the velocity with $r_{i}$.

For each single droplet, we use relativistic hydrodynamics and the equation of state of the entropy density [16, 42, 63, 64] to describe its subsequently evolution [28, 44]. The evolution of the granular source of many droplets can then be obtained by superposing all

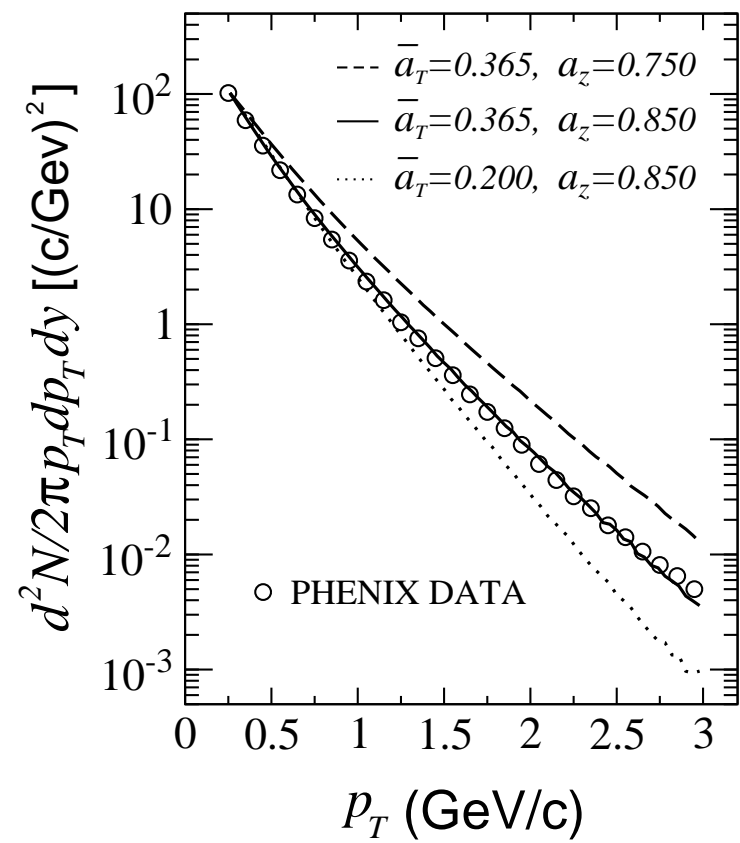

FIG. 3: Negative pion transverse momentum spectrum for $|y|<0.5$. The open circles give the PHENIX data for $\sqrt{s_{N N}}=200 \mathrm{GeV} \mathrm{Au}+\mathrm{Au}$ collisions with minimum bias [65]. Various curves give theoretical predictions with different parameters.

of the evolutions of individual droplets 28, 44]. In our calculations, the transition temperature and the transition temperature width are taken to be $T_{c}=165 \mathrm{MeV}$ and $\Delta T=0.05 T_{c}[16,28,42,44]$. The initial energy density of the droplets is taken to be $\epsilon_{0}=3.75 T_{c} s_{c}$, which is about two times of the density of quark matter at $T_{c}$ [16, 42]. The pions are emitted out of the surfaces of droplets at the freeze-out temperature $T_{f}=0.95 T_{c}$, with momenta obeying the Bose-Einstein distribution in the local frame at the temperature $T_{f}$.

In our model calculations there are five velocity parameters, $\left\{a_{x}, a_{y}, a_{z}, b_{T}=b_{x}=b_{y}\right.$, and $\left.b_{z}\right\}$, and four size parameters of the granular source, $\left\{\mathcal{R}_{t}, \mathcal{R}_{z}, \Delta \mathcal{R}_{t}\right.$, and $\left.r_{d}\right\}$. We find that the pion transverse momentum spectra and the elliptic flow $v_{2}$ are sensitive to the velocity parameters and insensitive to the size parameters. While our HBT results are sensitive both to the velocity parameters and the size parameters. We first determine the velocity parameters by comparing the pion transverse momentum spectra and the elliptic flow $v_{2}$ of our model calculations with experimental data. Then, we determine the source size parameters by comparing our results of HBT radii with experimental data.

Figure 3 shows the PHENIX $\pi^{-}$transverse momentum spectrum in $\sqrt{s_{N N}}=200 \mathrm{GeV} \mathrm{Au}+\mathrm{Au}$ collisions with minimum bias [65] and the transverse momentum spectrum obtained in our model calculations. The theoretical results have been normalized by matching the distribution to the first $p_{T}$ bin. In our calculations, we use a cut of particle rapidity $|y|<0.5$ in order to compare with 
experimental data, which have been normalized to unit rapidity [65]. Instead of the velocity magnitude parameters of $a_{x}$ and $a_{y}$, we can equivalently use their average $\bar{a}_{T}=\left(a_{x}+a_{y}\right) / 2$ and their difference $\Delta a_{T}=a_{x}-a_{y}$. The transverse momentum spectra depend on $\bar{a}_{T}$ and is independent of $\Delta a_{T}$. They also depend on $a_{z}, b_{T}$, and $b_{z}$. We shall show that the elliptic flow $v_{2}$ is very sensitive to $\Delta a_{T}, b_{T}$ and $b_{z}$. Good agreement with experimental data of transverse momentum spectra and the elliptic flow can be obtained concurrently by taking $b_{T}=0.42$, $b_{z}=0.03$ and $\Delta a_{T}=0.10$. After $b_{T}$ and $b_{z}$ are fixed, the parameters $\bar{a}_{T}$ and $a_{z}$ can be obtained by comparing the transverse momentum spectrum of the granular sources with experimental data 65]. We finally determine $\bar{a}_{T}=0.365$ and $a_{z}=0.850$ for our granular source.

\section{RESULTS OF THE ELLIPTIC FLOW}

The transverse momentum distribution of particles can be represented in the form $66,67,68$

$$
\frac{d^{2} N}{d p_{T}^{2} d \phi}=\frac{d N}{2 \pi d p_{T}^{2}}\left[1+2 \sum_{n} v_{n} \cos (n \phi)\right]
$$

where $p_{T}=\sqrt{p_{x}^{2}+p_{y}^{2}}$ is the transverse momentum of the particle, $\phi$ is its azimuthal angle with respect to the reaction plane, and the harmonic coefficients, $v_{n}$ are anisotropy parameters. The elliptic flow is defined as the second harmonic coefficient $v_{2}$, which describes the eccentricity of the particle distribution in the momentum space. We choose the direction of $x$-axis in the reaction plane and the direction of $y$-axis out of the reaction plane. We can express $v_{2}$ as

$$
v_{2} \equiv\langle\cos 2 \phi\rangle=\left\langle\frac{p_{x}^{2}-p_{y}^{2}}{p_{T}^{2}}\right\rangle .
$$

Figure 4 shows the PHENIX $v_{2}$ data as a function of $p_{T}$ for charged pions in $\mathrm{Au}+\mathrm{Au}$ collisions at $\sqrt{s_{N N}}=200$ $\mathrm{GeV}$ [10]. Also shown are the theoretical $v_{2}$ results in our granular droplet model. We use the same cut for particle pseudo-rapidity, $|\eta|<0.35$, in theoretical calculations, as in the experiment set-up [10]. In the region $p_{T}<2 \mathrm{GeV}$, the $v_{2}$ coefficient for a granular source increases with $p_{T}$ as in previous hydrodynamical calculations [35, 36, 69, 70, 71, 72, 73]. However, in the high $p_{T}$ region with $p_{T}>2.5 \mathrm{GeV}$, we find that the $v_{2}$ coefficient for a granular source with small $b_{z}$ decreases with $p_{T}$, which is different from previous hydrodynamical calculations [8, 9, 10, 11, 36, 69, 70, 73]. In the droplet-rest frame, the average transverse momentum of particles in the $x$ and $y$ directions, $\left\langle p_{x}^{\prime}\right\rangle$ and $\left\langle p_{y}^{\prime}\right\rangle$, are the same. In the laboratory frame, a elliptic flow arises from the anisotropic collective transverse boost of the droplet. For a very small $b_{z}$, the longitudinal velocity of droplets is almost constant in the source while the magnitude of the longitudinal boost velocity $a_{z}$ is very large (0.85) in

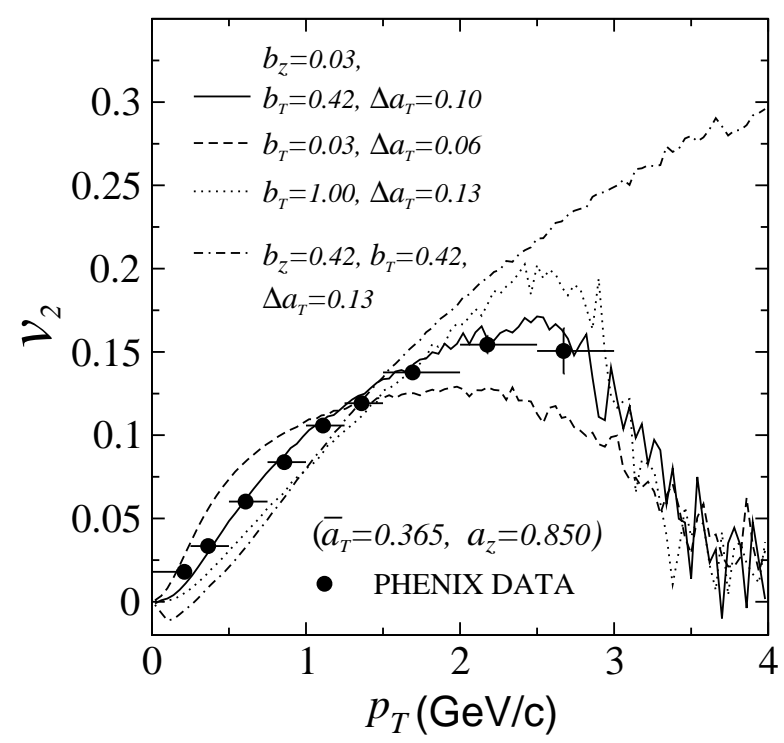

FIG. 4: Pion elliptic flow $v_{2}$ as a function of particle transverse momentum for the granular sources and the experimental data of charged pions of PHENIX [10].

our model. In this case, the longitudinal component of droplet velocity is important and it does not boost the transverse momenta of the particles. The transverse velocity of the droplet is much smaller than the longitudinal velocity of the droplet. Its boost effect becomes weaker for the particles with higher transverse momentum $p_{T}^{\prime}$ (higher $p_{T}$ ). On the other extreme for larger $b_{z}$, the longitudinal velocity of droplet is small for the droplets with small longitudinal coordinates. The transverse components of droplet velocity are dominant in the small $z$ region, which lead to a production of the elliptic flow and the $v_{2}$ coefficient increasing with transverse momentum 71], even at higher $p_{T}$ as shown in the dashed-dot curve in Fig. 4.

After $\bar{a}_{T}$ and $a_{z}$ have been fixed at the values 0.365 and 0.850 as determined by the pion transverse momentum spectra, we examine the pattern of $v_{2}$ as a function of transverse momentum $p_{T}$ as we vary $b_{T}, b_{z}$, and $\Delta a_{T}$. In the region of $p_{T}<2 \mathrm{GeV}$, the curvature of $v_{2}$ is sensitive to $b_{T}$. For $b_{z}=0.03$, the curvature for $b_{T}=0.03$ is too large (see the dashed line in Fig. 4) and the curvature for $b_{T}=1.00$ is too small (see the dot line in Fig. 4). For $b_{z}=b_{T}=0.42$, the curve of $v_{2}$ rises continuously and deviates from experimental data for $p_{T}>1.5 \mathrm{GeV}$. We find that the set of parameters $\left\{b_{z}, b_{T}, a_{T}\right\}=\{0.03,0.42,0.10\}$ give results consistent with experimental $v_{2}$ data, as indicated by the solid theoretical curves in Fig. 4. The fact that $b_{T}$ is much greater than $b_{z}$ while $a_{z}$ is substantially greater than $a_{T}$ indicates that the dynamical behavior in the transverse expansion and longitudinal expansion are very different.

Recent experimental data of the elliptic flow of pion show that $v_{2}$ remains to have the value of 0.1 at very high $p_{T}$ [74]. The processes of particle production in the 
high $p_{T}$ region are dominated by parton and hard-probe processes and is beyond the thermal emission model we consider here.

\section{RESULTS OF HBT INTERFEROMETRY}

The two-particle Bose-Einstein correlation function is defined as the ratio of the two-particle momentum distribution $P\left(p_{1}, p_{2}\right)$ relative to the the product of the single-particle momentum distribution $P\left(p_{1}\right) P\left(p_{2}\right)$. For a chaotic pion-emitting source, $P\left(p_{i}\right)(i=1,2)$, and $P\left(p_{1}, p_{2}\right)$ can be expressed as 75 .

$$
\begin{gathered}
P\left(p_{i}\right)=\sum_{X_{i}} A^{2}\left(p_{i}, X_{i}\right), \\
P\left(p_{1}, p_{2}\right)=\sum_{X_{1}, X_{2}}\left|\Phi\left(p_{1}, p_{2} ; X_{1}, X_{2}\right)\right|^{2},
\end{gathered}
$$

where $A\left(p_{i}, X_{i}\right)$ is the magnitude of the amplitude for emitting a pion with 4 -momentum $p_{i}=\left(\boldsymbol{p}_{i}, E_{i}\right)$ in the laboratory frame at $X_{i}$ and is given by the Bose-Einstein distribution with freeze-out temperature $T_{f}$ in the local rest frame of the source point. $\Phi\left(p_{1}, p_{2} ; X_{1}, X_{2}\right)$ is the two-pion wave function. Neglecting the absorption of the emitted pions by other droplets, $\Phi\left(p_{1}, p_{2} ; X_{1}, X_{2}\right)$ is simply

$$
\begin{aligned}
\Phi\left(p_{1}, p_{2} ; X_{1}, X_{2}\right) \\
=\frac{1}{\sqrt{2}}\left[A\left(p_{1}, X_{1}\right) A\left(p_{2}, X_{2}\right) e^{i p_{1} \cdot X_{1}+i p_{2} \cdot X_{2}}\right. \\
\left.\quad+A\left(p_{1}, X_{2}\right) A\left(p_{2}, X_{1}\right) e^{i p_{1} \cdot X_{2}+i p_{2} \cdot X_{1}}\right] .
\end{aligned}
$$

Using the components of "out", "side", and "long" [6. 77] of the relative momentum of the two pions, $q=\left|\mathbf{p}_{\mathbf{1}}-\mathbf{p}_{\mathbf{2}}\right|$, as variables, we can construct the correlation function $C\left(q_{\text {out }}, q_{\text {side }}, q_{\text {long }}\right)$ from $P\left(p_{1}, p_{2}\right)$ and $P\left(p_{1}\right) P\left(p_{2}\right)$ by summing over $\mathbf{p}_{1}$ and $\mathbf{p}_{2}$ for each ( $q_{\text {out }}, q_{\text {side }}, q_{\text {long }}$ ) bin [28]. The HBT radii $R_{\text {out }}, R_{\text {side }}$, and $R_{\text {long }}$ can then be extracted by fitting the calculated correlation function $C\left(q_{\text {out }}, q_{\text {side }}, q_{\text {long }}\right)$ with the following parametrized correlation function

$C\left(q_{\text {out }}, q_{\text {side }}, q_{\text {long }}\right)=1+\lambda e^{-q_{\text {out }}^{2} R_{\text {out }}^{2}-q_{\text {side }}^{2} R_{\text {side }}^{2}-q_{\text {long }}^{2} R_{\text {long }}^{2}}$.

Figure 5 shows the theoretical two-pion correlation functions for the granular source. The top figures give the results for a average pion transverse momentum of a pion pair, $K_{T}$, less than $400 \mathrm{MeV} / \mathrm{c}$, and the bottom figures for $K_{T}>400 \mathrm{MeV} / \mathrm{c}$. The velocity parameters of the granular source are $\bar{a}_{T}=0.365, \Delta a_{T}=0.10, a_{z}=0.850$, $b_{T}=0.42$, and $b_{z}=0.03$, which are determined by the transverse momentum spectra and the elliptic flow $v_{2}$ as discussed above. The size parameters of the granular sources are taken to be $\mathcal{R}_{t}=8.8 \mathrm{fm}, \Delta \mathcal{R}_{t}=3.5 \mathrm{fm}$, $\mathcal{R}_{z}=7.0 \mathrm{fm}$, and $r_{d}=1.3 \mathrm{fm}$. The number of droplet

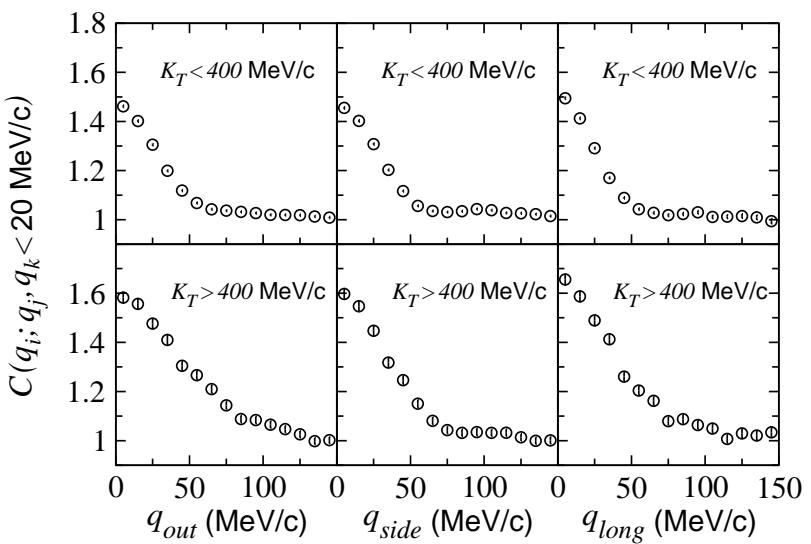

FIG. 5: Two-pion correlation functions for granular source.

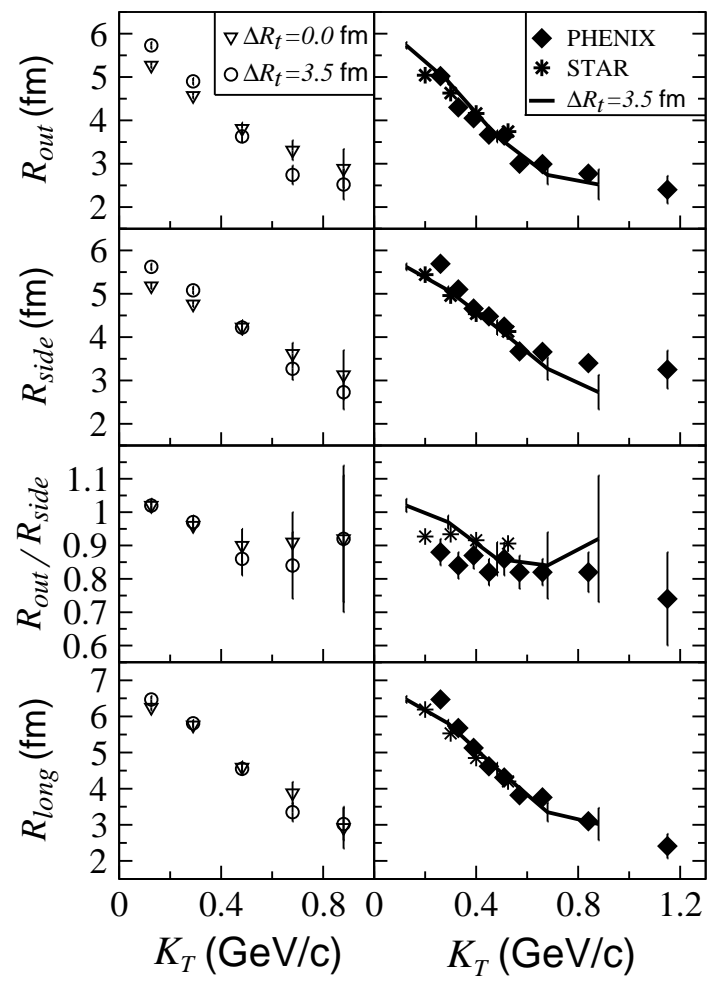

FIG. 6: Two-pion HBT radii obtained by the PHENIX Collaboration [14] and the STAR Collaboration 5] compared with the theoretical results calculated in the granular droplet model with $\Delta \mathcal{R}_{t}=0$ and $3.5 \mathrm{fm}$.

$N_{d}$ is taken to be 40 in our calculations. One observes noticeable differences in the lower $K_{T}$ region as compared to the higher $K_{T}$ region.

The left panels of figure 6 give the fitted two-pion HBT radii for the granular source as a function of $K_{T}$. The symbols of circle and down-triangle are for $\Delta \mathcal{R}_{t}=0.35$ fm and $\Delta \mathcal{R}_{t}=0$, respectively. The parameter $\Delta \mathcal{R}_{t}$ of Eq. (21) describes the radial distribution of the centers of the droplets, with $\Delta \mathcal{R}_{t}=0$ for a uniform distribution and $\Delta \mathcal{R}_{t}=0.35 \mathrm{fm}$ for a shell-type radial distribution. The experimental PHENIX results [14], and STAR re- 
sults [5] are shown on the right panels. The curve gives the theoretical results for $\Delta \mathcal{R}_{t}=0.35 \mathrm{fm}$. For our theoretical HBT calculations, we use a cut for particle pseudorapidity region $|\eta|<0.35$, the same as in the PHENIX experiments [14]. We find that if we increase the parameter $r_{d}$, the HBT radii $R_{\text {out }}$ and $R_{\text {long }}$ will increase. And if we increase the parameter $\Delta \mathcal{R}_{t}$, the variation of HBT radii $R_{\text {out }}$ and $R_{\text {side }}$ with $K_{T}$ will become steep. The HBT results of granular source for $\Delta \mathcal{R}_{t}=3.5 \mathrm{fm}$ agree quite well with experimental data, although the case with $\Delta \mathcal{R}_{T}=0$ also give almost as good an agreement.

\section{SUMMARY AND CONCLUSION}

The expansion of the dense matter produced in highenergy heavy-ion collisions may favor the production of granular droplets. The strong interaction associated with the dense matter will lead naturally to the presence of a surface tension on a boundary.

In an event-by-event basis, the initial transverse density distribution of matter in central nucleus-nucleus collisions have been known to be highly fluctuating 29, 41, 54]. This large spatial fluctuations may facilitate the formation of granular droplets. We envisage that as the expansion in the longitudinal direction proceeds much more rapidly than in the transverse direction, these initial transverse density fluctuations will lead to the formation of many longitudinal tubes of matter with a length much greater than its radius. Due to the surface tension effects, these tubes are unstable against granular sausage instability and will tend to break up into approximately equal-size spherical droplets.

Motivated by the possibility for the occurrence of granular droplets and our previous successful HBT analysis in terms of granular QGP droplets, we refine our previous granular QGP droplet model to include initial anisotropic velocities due to the anisotropic pressure gradients at an earlier stage of the collisions. We investigate concurrently the data of (1) the pion transverse momentum spectra, (2) the elliptic flow $v_{2}$ as a function of $p_{T}$, and (3) various $\mathrm{HBT}$ radii as a function of $K_{T}$, for $\mathrm{Au}+\mathrm{Au}$ collisions at $\sqrt{s_{N N}}=200 \mathrm{GeV}$ at RHIC. We find that the collection of different pieces of experimental data can be described well by a granular source model. Direct confirmation of the granular droplet configuration may need to await more experimental work on single-event HBT correlations and the fluctuation between correlation functions of the single- and mixed-events, as suggested previously [43, 44].

The extracted parameters from our analysis provide useful information on the nature of the transverse and longitudinal expansion. The elliptic flow $v_{2}$ is due to the anisotropic initial dynamical conditions in different transverse directions. Its decrease at high $p_{T}$ is sensitive to the exponential power parameter of the droplet velocity $b_{z}$ in the longitudinal direction. The value of $b_{z}$ is much smaller than that of $b_{T}$, indicating different dynamical behaviors in longitudinal and transverse directions. Our HBT results further indicate that the granular source has a small lifetime and a possible shell-type spatial distribution. Further studies on the origin of the difference in the transverse and longitudinal dynamics as well as the spatial distribution in the transverse direction will be of great interest.

\section{Acknowledgments}

W.N.Z. would like to thank Dr. V. Cianciolo and Dr. G. Young for their kind hospitality at Oak Ridge National Laboratory. We acknowledge discussions with Dr. T. Chujo, Dr. V. Cianciolo, Dr. B.-A. Li, and Dr. B. Zhang. This research was supported by the National Natural Science Foundation of China under Contracts No. 10275015 and No. 10575024, and by the Division of Nuclear Physics, US DOE, under Contract No. DEAC05-00OR22725 managed by UT-Battle, LC.
[1] M. Gyulassy and L. McLerran, nucl-th/0405013

[2] See also New Discoveries at RHIC: the current case for the strongly interactive QGP, RIKEN Scientific Articles, Volume 9, BNL, May 14-15, 2004.

[3] BRAHMS Collaboration, I. Arsene et al., Nucl. Phys. A 757, 1, 2005.

[4] PHOBOS Collaboration, B. B. Back et al., Nucl. Phys. A 757, 28, 2005.

[5] STAR Collaboration, J. Adams et al., Nucl. Phys. A 757, 102, 2005.

[6] PHENIX Collaboration, K. Adcox et al., Nucl. Phys. A 757, 184, 2005.

[7] STAR Collaboration, C. Adler et al., Phys. Rev. Lett. 87, 182301, 2001.

[8] STAR Collaboration, C. Adler et al., Phys. Rev. Lett. 89, 132301, 2002.

[9] STAR Collaboration, C. Adler et al., Phys. Rev. Lett.
90, 032301, 2003.

[10] PHENIX Collaboration, S. S. Adler et al., Phys. Rev. Lett. 91, 182301, 2003.

[11] STAR Collaboration, J. Adams et al., Phys. Rev. Lett. 92, 052302, 2004.

[12] STAR Collaboration, C. Adler et al., Phys. Rev. Lett. 87, 082301, 2001.

[13] PHENIX Collaboration, K. Adcox et al., Phys. Rev. Lett. 88, 192302, 2002.

[14] PHENIX Collaboration, S. S. Adler et al., Phys. Rev. Lett. 93, 152302, 2004.

[15] STAR Collaboration, J. Adams et al., Phys. Rev. C 71, 044906, 2005.

[16] D. H. Rischke and M. Gyulassy, Nucl. Phys A 608, 479 (1996).

[17] D. Teaney and E. Shuryak, Phys. Rev. Lett. 83, 4951 (1999). 
[18] U. A. Wiedemann and U. Heinz, Phys. Rept. 319, 145 (1999).

[19] S. Soff, S. A. Bass and A. Dumitru, Phys. Rev. Lett. 86, 3981 (2001).

[20] R. M. Weiner, Phys. Rept. 327, 249 (2002).

[21] S. Pratt, Nucl. Phys. A 715, 389c (2003).

[22] S. Soff, S. A. Bass, D. H. Hardtke, and S. Y. Panitkin, J. Phys. G 28, 1885 (2002).

[23] U. Heinz and P. Kolb, Nucl. Phys. A 702, 269 (2002).

[24] Zi-Wei Lin, C. M. Ko, and Subrata Pal, Phys. Rev. Lett. 89, 152301 (2002).

[25] D. Teaney, Nucl. Phys. A 715, 817 (2003).

[26] T. Csörgö and J. Zimányi, Acta Phys. Hung. New Series, Heavy-Ion Physics, 17, 281 (2003), nucl-th/0206051

[27] D. Molnár and M. Gyulassy, Phys. Rev. Lett. 92, 052301 (2004).

[28] W. N. Zhang, M. J. Efaaf, C. Y. Wong, Phys. Rev. C 70, 024903 (2004).

[29] O. Socolowski Jr., F. Grassi, Y. Hama, and T. Kodama, Phys. Rev. Lett. 93, 182301 (2004);

[30] C. Y. Wong, J. Phys. G 29, 2151 (2003); C. Y. Wong, J. Phys. G 30, S1053 (2004); C. Y. Wong, AIP Conference Proceedings, 828, 617 (2006), hep-ph/0510258

[31] J. G. Cramer, G. A. Miller, J. M. S. Wu, and J. H. Yoon, Phys. Rev. Lett. 94, 102302 (2005).

[32] M. A. Lisa, S. Pratt, R. Soltz, U. Wiedemann, Ann. Rev. Nucl. Part. Sci. 55, 357 (2005); nucl-ex/0505014

[33] S. Pratt and D. Schindel, nucl-th/0511010

[34] E. Frodermann, U. Heinz, M. A. Lisa, Phys. Rev. C 73, 044908 (2006).

[35] T. Hirano and K. Tsuda, Phys. Rev. C 66, 054905 (2002).

[36] P. Huovinen, Nucl. Phys. A 715, 299 (2003).

[37] T. Humanic, Nucl. Phys. A 715, 641 (2003); T. Humanic, Int. J. Mod Phys. E 15, 197 (2006).

[38] M. Csanád, T. Csörgó, B. Lr̈stad. and A. Ster, J. Phys. G 30, S1079 (2004); M. Csanád, T. Csörgó, B. Lr̈stad, and A. Ster, nucl-th/0510027

[39] Z. W. Lin, C. M. Ko, B. A. Li, B. Zhang, S. Pal, Phys. Rev. C 72, 064901 (2005).

[40] B. Tomášik, nucl-th/0509100

[41] Y. Hama, Rone P.G. Andrade, F. Grassi, O. Socolowski Jr, T. Kodama, B. Tavares, S. S. Padula, hep-ph/0510096

[42] D. H. Rischke, Proceedings of the 11th Chris Engelbrecht Summer School in Theoretical Physics, Cape Town, February 4-13, 1998, nucl-th/9809044

[43] C. Y. Wong and W. N. Zhang, Phys. Rev. C 70, 064904 (2005).

[44] W. N. Zhang, S. X. Li, C. Y. Wong, and M. J. Efaaf, Phys. Rev. C 71, 064908 (2005).

[45] E. Witten, Phys. Rev. D 30, 272 (1984).

[46] W. D. Myers and W. J. Swiatecki, Ann. Phys. 84, 186 (1974).

[47] P. Möller, J. R. Nix, W. D. Myers, and W. J. Swiatecki, Atom. Data Nucl. Data Tabl. 59, 185 (1995).

[48] K. Kajantie and H. Kurki-Suonio, Phys. Rev. D 34, 1719
(1986).

[49] L. P. Csernai and J. I. Kapusta, Phys. Rev. Lett. 69, 737 (1992).

[50] Y. Iwasaki et al., Phys. Rev. D 49, 3540 (1994).

[51] B. Grossmann and M. L. Lauren, Nucl. Phys. B 408, 637 (1993).

[52] B. Beinlich, F. Karscha and A. Peikerta Phy. Lett. B 390, 268 (1997).

[53] L. S. Kisslinger, S. Walawalkar, and M.B. Johnson, Phys. Rev. D 71, 065017 (2005).

[54] H. J. Drescher, F. M. Liu, S. Ostapchenko, T. Pierog, and K. Werner, Phys. Rev. C 65, 054902 (2002).

[55] L. D. Landau, Izv. Akad. Nauk. SSSR. ser. fiz. 17, 51 (1953); L. D. Landau and S. Z. Belenkii, Usp. Fiz. Nauk 56, 309 (1955).

[56] P. Steinberg, Nucl. Phys. A 752, 423 (2005).

[57] M. Murray, the BRAHMS Collaboration, J. Phys. G 30, S667 (2004).

[58] C. Y. Wong, Ann. Phys. (N.Y.) 77, 279 (1973).

[59] L. D. Landau and E. M. Lifshitz, Fluid Mechanics, (Pergamon, New York, 1959).

[60] C. Y. Wong, J. Math. Phys. 17, 1008 (1976); C. Y. Wong, J. A. Maruhn, and T. A. Welton, Nucl. Phys. A 253, 469 (1975); C. Y. Wong, T. A. Welton, and J. A. Maruhn, Phys. Rev. C 15, 1558 (1977).

[61] F. Karsch, E. Laermann, and A. Peikert, Nucl. Phys. B 605, 579 (2001).

[62] C. Y. Wong, J. A. Maruhn, and T. A. Welton, Phys. Lett. B 66, 19 (1977).

[63] J. P. Blaizot and J. Y. Ollitrault, Phys. Rev. D 36, 916 (1987).

[64] E. Laermann, Nucl. Phys. A 610, 1 (1996).

[65] PHENIX Collaboration, S. S. Adler et al., Phys. Rev. C 69, 034909, 2004.

[66] C. Y. Wong, Phys. Lett. B 88, 39 (1979).

[67] J. Y. Ollitrault, Phys. Rev. D 46, 229 (1992).

[68] S. Voloshin, Y. Zhang, Z. Phys. Rev. C 70, 665 (1996); hep-ph/9407282

[69] P. F. Kolb, J. Sollfrank, U. Heinz, Phys. Rev. C 62, 054909 (2000).

[70] P. F. Kolb, P. Huovinen, U. Heinz, H. Heiselberg, Phys. Lett. B 500, 232, (2001).

[71] P. Huovinen, P. F. Kolb, U. Heinz, P. V. Ruuskanen, S. A. Voloshin, Phys. Lett. B 503, 58, (2001).

[72] P. F. Kolb, R. Rapp, Phys. Rev. C 67, 044903, (2003).

[73] P. Huovinen, nucl-th/0305064

[74] P. R. Sorensen, nucl-ex/0510052

[75] C. Y. Wong, Introduction to High-Energy Heavy-Ion Collisions (World Scientific, Singapore, 1994), Chap. 17.

[76] S. Pratt, Phys. Rev. Lett. 53, 1219 (1984); S. Pratt, Phys. Rev. D 33, 72 (1986); S. Pratt, T. Csörgo, and J. Zimányi, Phys. Rev. C 42, 2646 (1990).

[77] G. Bertsch, M. Gong, and M. Tohyama, Phys. Rev. C 37, 1896 (1988); G. Bertsch, Nucl. Phys. A 498, 173c (1989). 